\title{
EDITORIAL
}

\section{Applications and New Developments of Magnetic Resonance Techniques in Soil Science}

In soil and environmental sciences, magnetic resonance techniques are nowadays used for a large variety of applications such as those related to the characterization of natural organic matter, to the analysis of the interactions of pollutants with organic and inorganic moieties in environmental compartments, and to the evaluation of fluid flow in porous media. Recent advances in electronics allowed not only the development of NMR on solids and semi-solid samples, but also the evolution of in situ NMR equipments. In addition to classical NMR applications, magnetic resonance imaging (MRI) of soil-water and tracer transport processes in soil (e.g. spatial and temporal changes of soil-water distributions or the movement of wetting fronts, root growth and water uptake) and NMR relaxometry and diffusometry for pore space exploration in natural porous media are now important fields in soil studies.

This special issue includes the conference proceedings of the session SSS23 titled "Applications and new developments of magnetic resonance techniques in soil science" which was held in April 2009 in Vienna (Austria) within the Soil System Sciences program of the European Geoscience Union (EGU) General Assembly (http://meetingorganizer.copernicus.org/ EGU2009/session/947). During this first session in the EGU Assemblies dealing with NMR, all the aspects of NMR spectroscopy were accounted for: from the limits of the classical NMR techniques such as CPMAS ${ }^{13} \mathrm{C}$ NMR spectroscopy applied to soils and humic substances, to the developments of innovative NMR techniques for soil science investigations such as low field and relaxometry NMR.

The paper selection encompassed in the present special issue of The Open Magnetic Resonance Journal reflects the diversity of magnetic resonance applications in the field of soil and environmental sciences. It does of course not claim completeness in these fields, but is meant to give an interesting and hopefully inspiring excerpt of these continuously expanding research fields. The first part of the issue presents new developments and applications of low field NMR and relaxometry, followed by a paper on magnetic resonance imaging applied to the analysis of plant-soil interactions. The last part of the special issue concludes with the classical high field solid and liquid state NMR spectroscopy. Namely, a paper on the evaluation of the limits of CPMAS ${ }^{13} \mathrm{C}$ NMR spectroscopy due to imperfect field homogenization along the coil is presented together with a second paper, where interactions among a prion protein and a soil-like system were investigated. Finally, the last paper deals with the suggestion of a quick method for validity evaluation of the humic substances models which were suggested in the literature.

\section{Pellegrino Conte}

Università degli Studi di Palermo

Dipartimento di Ingegneria e Tecnologie Agro-Forestali

v.le delle Scienze 13, edificio 4

90128, Palermo

Italy

E-mail: pellegrino.conte@ unipa.it

\section{Andreas Pohlmeier}

Institute of Chemistry and Dynamics of the Geosphere

ICG-4: Agrosphere

Forschungszentrum Jülich $\mathrm{GmbH}$

52425 Jülich

Germany

E-mail: a.pohlmeier@fz-juelich.de
Anne E. Berns

Institute of Chemistry and Dynamics of the Geosphere ICG-4: Agrosphere Forschungszentrum Jülich GmbH

52425 Jülich

Germany

E-mail: a.berns@fz-juelich.de

Giuseppe Alonzo

Università degli Studi di Palermo Dipartimento di Ingegneria e Tecnologie Agro-Forestali v.le delle Scienze 13, edificio 4 90128, Palermo Italy E-mail: alonzo@unipa.it

\footnotetext{
(C) Conte et al.; Licensee Bentham Open.

This is an open access article licensed under the terms of the Creative Commons Attribution Non-Commercial License (http://creativecommons.org/licenses/by-nc/3.0/) which permits unrestricted, non-commercial use, distribution and reproduction in any medium, provided the work is properly cited.
} 